Techniques \& Culture

\title{
La mort du tambour
}

The Death of the drum

\section{James Leach}

\section{OpenEdition}

Journals

Édition électronique

URL : https://journals.openedition.org/tc/6229

DOI : $10.4000 /$ tc. 6229

ISBN : 1952-420X

ISSN : 1952-420X

\section{Éditeur}

Éditions de l'EHESS

Édition imprimée

Date de publication : 15 juin 2012

Pagination : 28-47

ISBN : 2-7351-1512-7

ISSN : 0248-6016

\section{Référence électronique}

James Leach, « La mort du tambour», Techniques \& Culture [En ligne], 58 | 2012, mis en ligne le 07 décembre 2012, consulté le 29 septembre 2022. URL : http://journals.openedition.org/tc/6229 ; DOI : https://doi.org/10.4000/tc.6229 


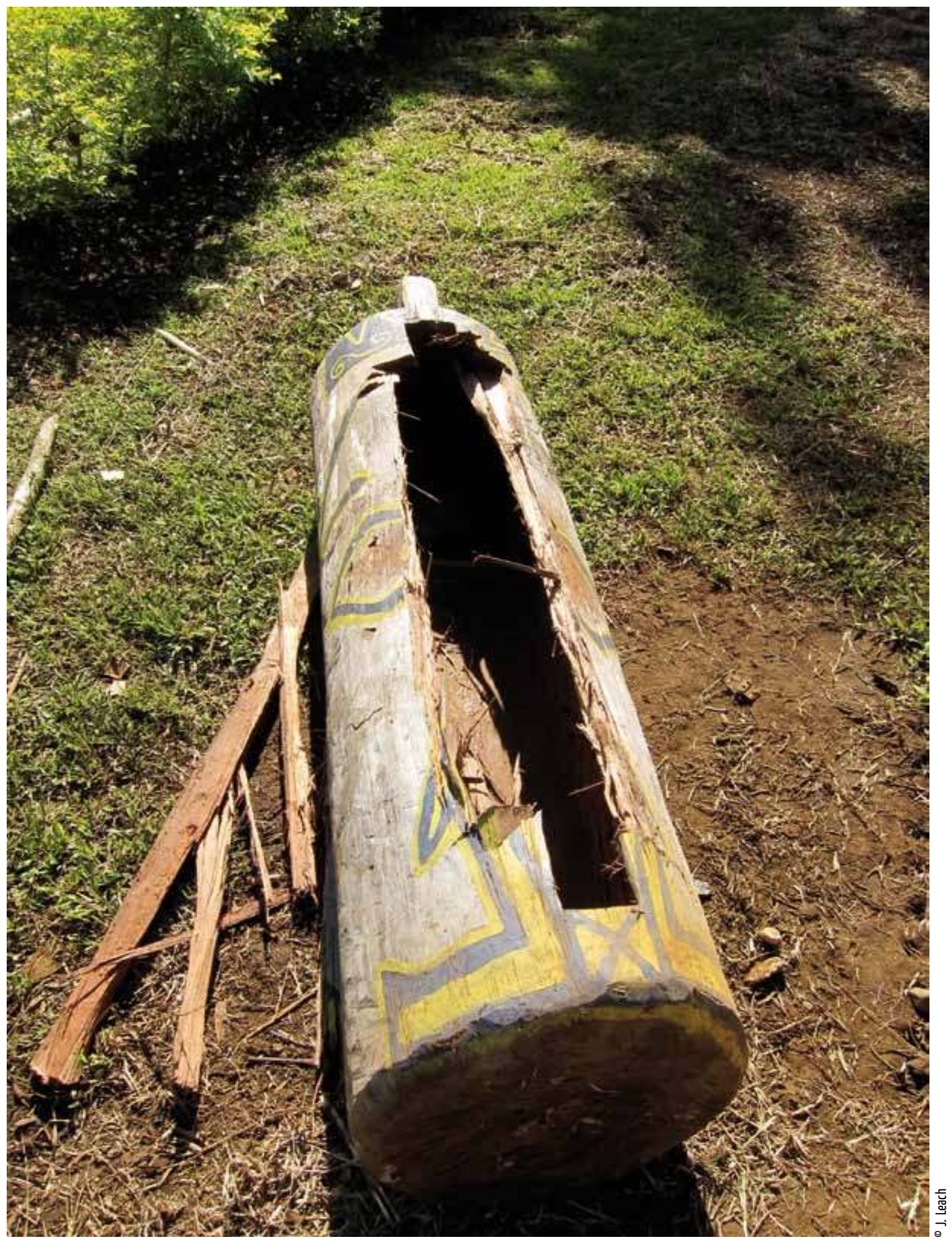


James Leach

Université d'Aberdeen. Département d'anthropologie. james.leach@abdn.ac.uk
Objets irremplaçables - I

Techniques \& Culture 58, 2012/1: 28-47

\title{
LA MORT DU TAMBOUR
}

\author{
Choses uniques sur la Rai Coast \\ de Papouasie-Nouvelle-Guinée
}

\section{La substitution et les formes irremplaçables}

Cet article se divise en deux parties. Dans la première, j'entends montrer comment un grand tambour à fente pourrait raisonnablement être considéré comme « irremplaçable » pour ceux qui le fabriquent et l'utilisent, malgré la possibilité pratique de son remplacement. Dans la seconde, je me pencherai sur un incident où il apparaît que la relation à de telles choses change avec l'arrivée de nouveaux types d'institution et de manières de relier les gens entre eux, ainsi que les personnes et les objets, manières qui correspondent à ces institutions. Ces événements se déroulent sur la Rai Coast de Papouasie-NouvelleGuinée. Dans la première partie de l'article, je me réfère sans vergogne à une analyse antérieure que j’ai développée à propos des tambours à fente. En revisitant cette analyse, je présenterai le cas de quelque chose qui est considéré comme irremplaçable par les personnes qui le fabriquent et l'utilisent. Puis je raconterai un événement sans précédent qui permet d'illustrer dans quelles conditions on est amené à penser le contraire. En l'occurrence, pour la première fois... (à la connaissance de la population locale) un tel objet fut «attaqué » et « tué ». Je replace cette «attaque » dans son contexte - à savoir le statut spécial de ce tambour à fente particulier. J'émets l'idée que le tambour attaqué constitue le premier « simulacre » d'un tambour à fente fabriqué par cette population: un objet reproductible ou générique, parce qu'il représentait un type de personne, alors que les «vrais » tambours à fente sont irremplaçables, car ils sont ce qui détermine l'apparition unique, qui se produit une fois seulement, d'autres personnes également 
uniques. Les relations qui s'étaient créées autour du tambour qui subit l'attaque en firent un objet générique plutôt qu’une chose personne unique.

Afin de comprendre ce qui rend quelque chose unique et donc irremplaçable (si une telle chose existe), nous devons examiner ce qu'implique la substituabilité. Avec sa longue histoire de travaux ethnographiques démontrant la place centrale de l'échange (une forme de substitution), la Mélanésie est sans aucun doute une région propice à l'exploration de cette question (ex.: Weiner 1992, Foster 1995). Dans le cas que je présente ici, je prends l'exemple de mes informateurs de Reite, un village dans l'arrière-pays de la Rai Coast, dans la Province de Madang, et je m'intéresse à un objet fabriqué particulier: un tambour à fente considéré comme une sorte de personne. Les tambours à fente sont fabriqués et utilisés en nombre par les populations de langue austronésienne, et par d'autres qui se sont adaptées à leur usage ou bien l'ont adopté après des contacts lors d'échanges commerciaux. Bien que les villageois de Reite parlent une langue papoue appelée Nekgini (Wurm \& Hattori 1981), ils fabriquent et utilisent ces étonnants objets sur la Rai Coast. Dans les analyses antérieures mentionnées plus haut, j'ai montré comment il est possible d'interpréter la fabrication de ces tambours à fente comme la quintessence des moments de production et de reproduction de la parenté et de la politique de Reite. Les habitants de Reite parlent et agissent lorsqu'ils sont à proximité des tambours à fente comme si ceux-ci étaient des personnes (sexuées). Mais l'idée de substituabilité nous invite à prêter attention au fait que les tambours ne sont pas identiques aux autres types de personnes de Reite. Dans les lignes qui suivent, j'établis un lien entre cette version d'une question ancienne concernant la substituabilité et la notion d'irremplaçabilité. Qu'est-ce qui rend une chose irremplaçable?

Les récentes analyses de matériaux mélanésiens indiquent et soulignent souvent le statut similaire des personnes et des choses, ou comment les choses peuvent être des personnes, et partant, comment elles peuvent avoir une sorte d'« agentivité ». De nombreux Mélanésiens peuvent faire et font comme si les choses étaient des personnes, tout en sachant parfaitement qu'il existe des différences entre ces choses-personnes et des personnes en tant qu'elles sont des personnes. Le problème que cela pose pour notre compréhension est récurrent, et la solution ne me semble pas consister à suivre Gell en envisageant une sorte d'agentivité secondaire, ou médiatisée pour les objets (voir Gell 1998, Leach 2007, 169). Je m’oppose à Gell en partie parce qu'agir de la sorte repose sur une théorie de la représentation attribuée aux sujets ethnographiés: le fait que les objets représentent des gens pour eux, comme dans cette idée de Gell selon laquelle ils sont des index de l'intentionnalité de leur créateur. En adoptant une telle analyse, l'anthropologue ne prend peut-être pas assez en compte tous ces endroits et gens qui revendiquent le statut de personnes pour des choses, ni la capacité que l'on attribue à ces objets d'agir, non comme des représentations d'autres personnes mais, comme des personnes de plein droit. Autrement dit, l'approche en termes d'« agentivité secondaire» remplace ce que les gens font et disent par une version qui, de fait, explique et donc explicite leurs déclarations sans leur laisser leur pleine puissance d'action et de pensée. Mon objectif n'est pas de discuter l'agentivité ni de prouver qu'une chose peut être une personne, mais de montrer où et comment des choses qui sont faites objets sont tout à la fois comme des personnes et pas comme des personnes, et partant irremplaçables. J'ai pris au sérieux la tâche de comprendre ce qui fait d'une chose une personne à Reite, en assistant aux moments où ils lui accordent une personnalité entière, et aux moments où il est évident pour eux que la chose possède un type de présence différent des personnes humaines. Leur distinction ne se fait pas entre un type d'agentivité primaire et secondaire, mais entre, 
d'une part des choses qui sont placées entre des personnes, des choses qui expriment leur différence et leur association en tant qu'entités actives, et, d'autre part des objets qui jouent le rôle de quelque chose qui, sinon, serait absent. Les deux types de chose/ objet influencent la forme des relations sociales dans leur « environnement proche » (pour reprendre l'expression de Gell), mais le second le fait en suscitant la mémoire ou l'affect, tandis que le premier participe à la transformation des relations entre personnes, et donc à la constitution même de ces personnes.

Ces considérations (qui passent par la description précise de la logique et des pratiques à propos des choses dans leur relation aux personnes sur la Rai Coast) conduisent à se demander dans quelle mesure une personne est remplaçable, comment on peut remplacer une chose par une autre - une personne par une effigie, ou une voix par un tambour. Comme je l'ai dit précédemment, ce sont là des questions classiques pour un spécialiste de la Mélanésie. En conclusion, je suggère que l'attaque inédite envers le tambour à fente a été rendue possible par le fait qu'il était, d'une certaine manière, une chose nouvelle pour ces personnes: un tambour à fente générique, un tambour ordinaire - pas un « vrai » garamut au sens où nous l'entendons, tant moi-même dans mon analyse d'ethnologue, que les habitants de Reite. C'est-à-dire que le tambour brisé n'avait pas été créé avec succès comme un type de personne, et n'était donc pas «irremplaçable ». L'article ajoute donc des éléments nouveaux aux analyses antérieures, puisqu'il aborde la question de savoir ce qui fait d'un tambour une personne, mais aussi ce qui fait qu'il n'en est pas une. La notion d'irremplaçabilité invite aussi à examiner celles d'essence et d'identité. De nombreuses analyses de données récoltées en Mélanésie démontrent que les choses et les personnes ne sont pas définies par quelque essence éternelle et interne, mais par leur participation à des moments, et en tant que moments (ce que Strathern appelle « réifications » $(1988: 177)$ dans des processus « sociogéniques » (Pfaffenberger 1992 : 492). Je me pencherai donc également sur la temporalité de la personnalité (au sens de ce qui constitue une personne), dans sa forme spécifique à Reite.

Un tambour à fente (garamut en Tok Pisin, la lingua franca néo-mélanésienne) est un idiophone. On en retire un son en frappant la surface extérieure du tronc évidé. Les garamut émettent un profond son de stentor qui résonne et porte sur de longues distances. Ils sont utilisés quotidiennement pour envoyer des messages entre les hameaux, dans l'environnement accidenté et pentu de la forêt pluviale de la Rai Coast, et en particulier à Reite, qui est situé à 500 mètres au-dessus du niveau de la mer et à 7-10 kilomètres à l'intérieur des terres. Les messages consistent en une série de battements codés. Les possibilités de « dire » des choses par le truchement d'un garamut sont innombrables. Les habitants de Reite m'ont souvent dit, pour plaisanter, qu'il s'agissait d'un équivalent papou du téléphone.

À Reite, un garamut est considéré comme quelque chose de spécial parce qu'il dispose d'une voix. Il peut parler pour son propriétaire d'une façon qui serait impossible sans sa présence physique. Cela paraît évident, mais je vais montrer que c'est vrai dans un sens original. Ce qui est « fait » dans la fabrication d'un garamut est à la fois la possibilité de parler d'une façon particulière (à distance en utilisant un tronc évidé, etc.) et les conditions dans lesquelles le bruit que produit le tronc évidé peut être, et est considéré comme une voix mais aussi ce qui est nécessaire pour que des personnes identifient ce bruit comme la voix et les intentions d'une personne spécifique.

Chaque homme initié de Reite possède un nom de tambour à fente, c'est-à-dire une séquence unique de battements qui lui est donnée par le frère de sa mère au cours de 
Takarok avec une igname, 2006

(Fig. 2)

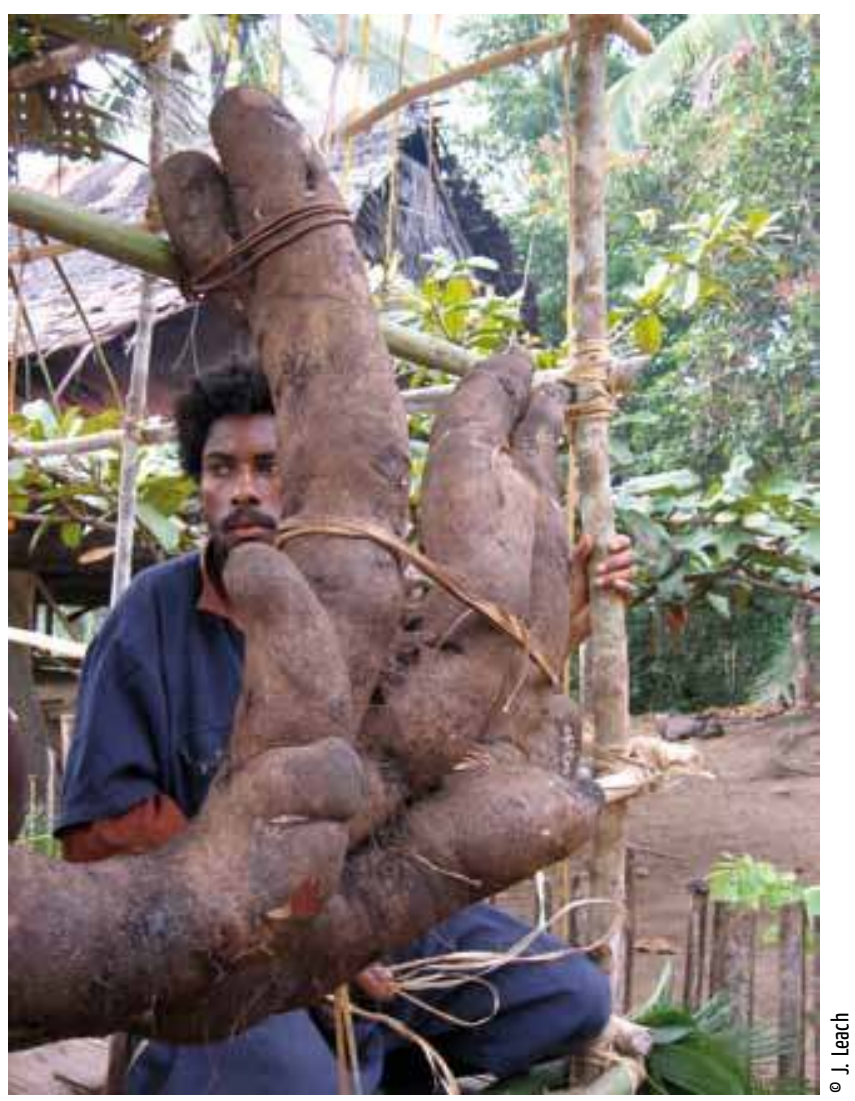

son initiation. En fait, cette séquence est empruntée au répertoire de musique sacrée du frère de la mère. Dans l'article que j'ai publié dans le Journal of Royal Anthropological Institute en 2002, j’ai examiné la fabrication de ces objets, à partir des prémisses locales les concernant, fréquemment exprimées par les habitants de Reite, selon lesquels un garamut « est » un homme (Leach 2002). Il a explicitement le statut d'une personne, et parce qu'il a une voix, il doit être traité avec respect.

On pourrait ajouter qu'un garamut est également une partie d'une autre personne, étant donné que l'homme pour lequel il a été fabriqué est étroitement associé à son apparence et au son qu'il produit. L'analyse de la première partie de l'article se focalisait sur le fait qu'un garamut est fabriqué pour un homme adulte par ses affins. Donc, en même temps qu'il reçoit son « signal d'appel » (Burridge 1960) de la part du frère de sa mère (une personne séparée géographiquement ainsi qu'en termes de catégorie dans ce système marital virilocal), il reçoit le garamut lui-même d'un tel parent séparé - d'affins qui habitent un autre endroit.

On a observé depuis longtemps qu'en Mélanésie les personnes sont considérées comme pouvant prendre des différentes formes, ou que des choses peuvent prendre des formes de personne. On le constate depuis les travaux de Reo Fortune sur les ignames de l'île de Dobu (Fortune 1932) jusqu'à ceux de Gudeman sur la politique économique à l'œuvre derrière la logique des Dobuan (1986), en passant par la puissante conceptualisation du genre par Strathern (1988), en tant qu'idiome dans lequel les formes des personnes sont reconnues, ou encore dans les travaux de Damon (1980 : 284). À ce propos, Marilyn Strathern écrit (de façon symptomatique (?) que «nous devons garder à l'esprit que les personnes objectifient des relations; en conséquence de quoi les corps et les esprits sont leur manifestation réifiée. Et elles doivent toujours prendre une forme manifeste, c'est-à-dire exhiber une condition différenciée » (1988: 299).

Les personnes et, assurément, les corps, sont composés d'autres corps et de nourriture. Le mythe d'origine de l'igname rapporte comment un homme maltraité par ses cupides enfants et petits-enfants s'est un jour transformé en igname et leur a dit de le manger pour se nourrir après sa mort. Son nom (secret) est l'élément central des pratiques indispensables pour que les jardins d'ignames soient prospères. Il ne s'agti pas ici d'une erreur de catégorie de la part des habitants de Reite. Exactement comme « les Dobu ne sont pas embarrassés par la différence entre les ignames et les humains; le fait est que les personnes du lignage peuvent prendre le corps de l'un ou l'autre type d'êtres » (Strathern 1995 : 17).

Certes, les ignames sauvages poussent dans la jungle, mais celles qui poussent dans des jardins sont différentes: ces dernières sont déjà anticipées comme une part future des corps socialement produits des autres. Comme l'écrit Coupaye, « les choses ne sont pas simplement consommables, il est fait en sorte qu'elles le soient » $(2009$ : 93, 106). Les gens 


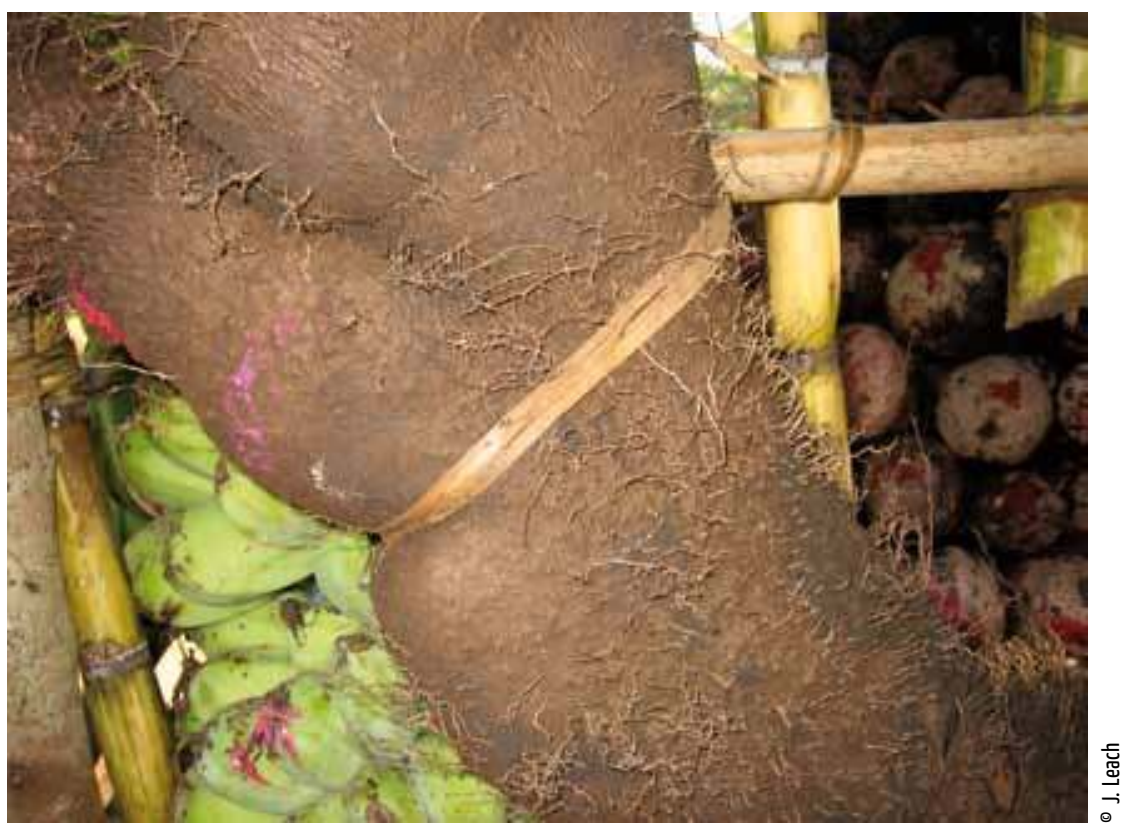

La peau de l'igname, 2006

La figure montre une igname de Reite, débarrassée de sa couche de terre et faisant partie d'un présentoir considéré en lui-même comme une effigie. Notez la qualité de la peau, l'aspect absolument « humain » de la chose. S'il n'y a pas de correspondance simple entre cette perception profonde d'une « humanité » de l'igname et ce que les habitants de Reite pensent, il n'en demeure pas moins que les ignames sont pour eux une sorte de personne.

(Fig. 3)

savent pour qui on fait pousser les ignames, quels corps elles vont contribuer à former. Leur croissance dans la terre est une manifestation du travail de quelqu'un travaillant pour quelqu'un d'autre, leur succès indique la condition morale et rituelle du cultivateur, et leur production contribue à la création d'un personnage et d'une réputation par référence auxquels d'autres gens adaptent leur propre travail et leurs propres actions à proximité de ce producteur. On fait pousser les ignames pour d'autres que soi - elles font partie d'échanges cérémoniels plus larges dans lesquels les corps sont remplacés par d'autres corps. Ces échanges ne sont pas des événements vides, ils ne sont pas effectués pour le simple fait de la « tradition »; ils continuent d'être les façons nécessaires et vitales dont la reproduction se passe à Reite.

Jusqu'à l'arrivée des cultures commerciales (dans les années 1980-1990) et l'introduction, bien plus récente, de marchés pour les produits des jardins (voir Leach 2011 : 302-308), l'ensemble de l'horticulture et de l'élevage était orienté vers ces cycles de croissance, d'échange et de reproduction. Tous les jardins sont cultivés dans le but de donner leur produits à d'autres, en retour des corps que l'on reçoit. Ainsi, pour ces gens, l'igname est à l'image de, réagit à, et constitue d'autres personnes.

La figure 4 montre un palem, une effigie constituée de produits horticoles dont la décoration rappelle celle des personnes. Sur les figures 6 et 7, des gens de Reite extériorisent l'intérieur d'eux-mêmes (cf. Strathern 1979: 254), la peau peinte d'ocre rouge, portant des ornements en dents de chien et des richesses en coquillage qui illustrent la force et la persistance des os. Ces « os » peuvent être détachés de la personne. Ils peuvent être donnés, et sont donnés en combinaison avec la nourriture des jardins afin de remplacer les corps d'autres personnes. C'est là ce que disent les gens de Reite à propos du palem. La nourriture cultivée et la viande d'animaux élevés sont non seulement préparées pour être offertes aux autres dans les cycles d'échange reproductif, mais le palem est également accepté comme remplacement de la substance du corps donné lors du mariage. Pour 

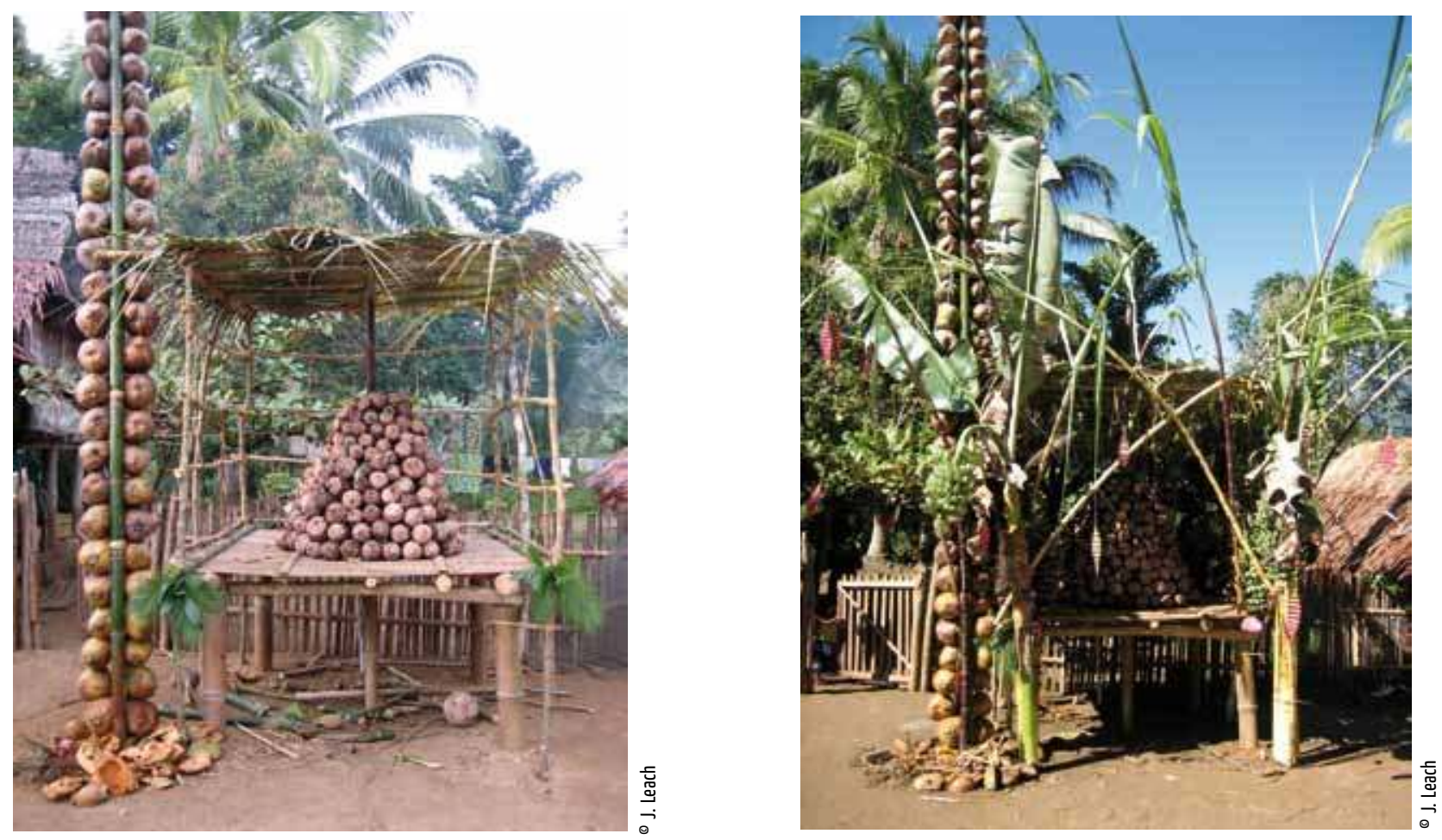

\section{Une pile de tarossur un palem.}

La Figure 4 montre à nouveau de la nourriture tirée des jardins (ici des taros). Les tubercules sont empilés sur une construction où des ignames sont aussi exposées, construction explicitement nommée « effigie ». Elle est dotée d'une colonne vertébrale, de côtes, d'un visage et de parties internes - c'est-à-dire des viscères, le taro lui-même qui constituent la substance du corps (Leach 2003 : 162-168).

L'ensemble, qui porte le nom de palem, est explicitement et consciemment organisé tel un corps, puisqu'il est la pièce centrale de ce qui est montré puis offert en retour d'un autre corps - celui d'une femme à épouser, et du premier enfant (dû à sa parentèle de naissance).

(Fig. 4 et 5) reprendre les termes de Strathern, le corps est constitué de, et déjà dû à « des relations aux autres », dont il est une "manifestation réifiée ». Son apparence de chose - en tant qu'igname, palem, enfant ( $c f$. Figure 8), ou adulte humain - manifeste sa condition différenciée. Le corps, tout comme la personne, n'est jamais achevé, il est sans cesse nourri et soigné, et protégé, et lui-même éduque, prodigue des soins et protège -, donnant aux autres et recevant de leur part, et se transformant au cours de ce processus.

Si les relations aux autres se manifestent sous la forme du corps, alors la spécificité de ces relations est claire sous cette forme et cette apparence. Un jeune marié (Figure 6) a des relations aux autres qui diffèrent de celles qu'ont son épouse, son beau-père, ou sa fille, ou, en vérité, l'igname (en tant qu'objet pris dans un réseau de relations) qu'il a fait pousser (Figures 8 et 2). Son apparence - ce qu'il est bon pour lui de montrer - reflète sa position.

Les gens de Reite effectuent des transformations de statut par, et lors de leurs échanges cérémoniels. Ils le font également d'autres manières, dont la plus importante pour un homme est sa réception d'un garamut - qui est seulement fabriqué pour lui être offert à un moment précis de son cycle de vie : lorsqu'il s'est marié et a rempli les principales obligations du mariage. C'est-à-dire lorsqu'il a remplacé ou acheté le « corps » de son épouse auprès de sa parentèle affine par des échanges qui remplacent explicitement le corps de l'épouse apporté sur les terres du mari par un autre corps : cette effigie d'un corps faite de produits du jardin, d'un porc, de richesses, etc., appelée palem. Bien sûr, pour atteindre ce stade, l'homme doit être déjà bien avancé dans les obligations à remplir par des échanges cérémoniels. Pour se marier, il doit avoir été initié et être parvenu à payer cette transformation de son corps par le frère de sa mère par le biais d'un échange conséquent et astreignant. En fait, chaque moment du développement de la personne à Reite rend visible des relations particulières et pertinentes avec d'autres personnes, au même moment et en même temps que ces relations sont transformées par le biais de la substitution (voir Leach 2003 : 140, 148, 151). Le moment où l'on fabrique un garamut pour un homme est considéré comme un 
point culminant de son émergence en tant qu'homme (ayant désormais un garamut) doté de présence et d'autorité. C'est-à-dire qu'elle est rendue spectaculairement apparente, visible, non seulement par l'objet lui-même, mais aussi par la capacité de celui-ci à « parler » au loin, à avoir sa voix « entendue » par les autres.

C'est sur le dernier aspect de ce processus que j'ai porté mon attention dans mon article plus ancien (Leach 2002 : 278-279). C'est-à-dire que, tandis que la fabrication du garamut requiert clairement d'être compétent dans ce travail, c'est la monstration et la mise en œuvre des relations politiques et de parenté liées à sa fabrication et à son apparence qui reçoivent le plus d'attention.

Les garamut sont fabriqués par les hommes, isolés en secret, lors du culte masculin. Les affins de l'homme à qui est destiné le garamut viennent avec leurs esprits sur ses terres, où ils coupent un arbre. On dit que ce sont les esprits qui transforment le tronc d'arbre en une série de garamut, le commanditaire (sponsor), la « base » ou « raison » de ce travail prenant le plus grand, fait avec la base du tronc. Ses frères peuvent en extraire d'autres plus hauts dans le tronc, ces garamut/voix étant soutenus par sa position fondamentale (en tant que sponsor) vis-à-vis de ses affins et se reposant sur elle. Notons que les germains ne peuvent bien sûr prendre leur propre garamut, et ainsi obtenir une « voix » propre que si leurs propres affins veulent bien venir et entreprendre la transformation d'une section du tronc pour eux. Ils doivent aussi leur payer ce qu'il faut. Tous les frères sont sous l'autorité de l'homme qui a prévalence dans la lignée, de sa voix. Une position d'autorité sur les germains est rendue présente et réelle par l'apparition des garamut en tant qu'ils sont des manifestations de l'alliance à travers le mariage que l'homme de « base » a contractée.

C'est en «mangeant» leur partie interne que les esprits transforment les troncs en garamut - et ce processus est absolument analogue à celui par lequel les garçons cachés en forêt sont «mangés » par les esprits du frère de la mère lors des initiations, vêtus de parures sur leur lieu d'isolement, et finalement réapparaissent après leur transformation pour être vus « pour la première fois » en tant qu'hommes nouveaux.

Lorsque les habitants de Reite parlent du garamut comme d'une personne, comme d'une partie d'une personne, et comme de la voix d'une personne, ils font référence à cet entremêlement complexe de l'émergence d'un objet physique accompagnant de façon inséparable celle d'un homme en tant qu'homme particulier, situé dans un paysage de parents et de relations affines. Il se trouve dans une position et doté d'une présence dont il ne disposait pas avant la fabrication du garamut. La « voix » du garamut n'est pas simplement le son qu'il produit - qui est fort et clair -, mais, ce qui est plus important est que ce son est entendu comme la voix d'un homme précis, et qu'il y a des gens qui lui répondent comme ils répondent à la voix d'un homme duquel ils sont les obligés, du fait du processus même de fabrication du garamut (c'est-à-dire du fait même qu'ils se sont rassemblés pour le fabriquer en premier lieu, et qu'ils aient reçu des richesses conséquentes pour leur peine). Le garamut a une voix parce qu'il a été fabriqué pour être entendu.

\section{La non substituabilité et la stabilité temporelle d’un statut}

Bien sûr, de nombreux hommes possèdent un garamut. Leur aspect «irremplaçable » ne découle donc pas de leur rareté. Il provient du fait qu'ils sont uniques dans un sens 
bien plus important. Ils ont une position unique en tant que pivot et générateur d'une série de relations entre des personnes qui sont uniques. En ce sens, ils constituent bien des « personnes ». C'est-à-dire que, si une personne constitue un point unique dans une série de relations entre d'autres personnes, et si elle tire son identité, ses compétences, son savoir et son apparence, etc. de cette position unique, alors, un garamut est similaire à une personne. Il a une identité et un effet de par la position relationnelle qu'il occupe. Tout le monde est une personne. Mais ça ne signifie pas que les personnes peuvent être remplacées les unes par les autres, au premier sens de « remplacer». Chaque personne est unique, et c'est là que la prise en compte de quelque chose dans son unicité, en tant que chose unique, devient si intéressante. En l'occurrence, je vais tenter de démontrer que les personnes et les garamut sont également différents selon cette logique, et de dire pourquoi. Cette démonstration fait référence à l'apparence matérielle des relations selon des formes différentes, des formes différentes de réification à des moments précis.

Les personnes sont uniques, et pourtant, comme nous l'avons vu, elles sont substituables. En fait, un garamut n'est pas si difficile à remplacer (en tant que tronc creusé), mais il n'est pas substituable. En d'autres termes, il existe ici un entremêlement entre personne et objet qui les rend similaires et pourtant différents.

En 1995, j'ai eu l'occasion de rassembler une collection d'objets provenant de Reite pour le National Museum de Port Moresby (PNG), et pour le British Museum de Londres. J'ai bien sûr souhaité y inclure un garamut, objet le plus grand et le plus important de la culture matérielle locale, mais il s'est vite avéré que je ne pouvais pas obtenir un garamut existant déjà. La situation était simple: personne n'était prêt à vendre le sien.

J'ai déjà parlé des effigies (palem) données en remplacement des corps de la région de la Rai Coast. Cela démontre indubitablement que les corps y sont substituables et échangeables. En fait, la personne en tant que lien et point de rencontre entres des relations particulières n'est qu'une manifestation de ces relations, elle n'en est qu'une objectification à un moment donné. Ces relations changent naturellement et nécessairement au long d'un cycle de vie, si bien que la substance constitutive de la personne (par ex. l'origine de la nourriture que l'on consomme pour entretenir son corps, en tant qu'elle résulte d'un travail particulier, ou bien les parures que l'on porte sur la peau) est remplacée à chaque étape par différents éléments (qui proviennent de différentes sources). Construire des corps à partir d'une série de relations (avec la terre où sont établis des jardins, avec des esprits, avec les parents de son groupe de naissance) produit un corps différent de celui construit à partir d'autres relations (voir Biersack 1982 à propos des relations qu'établissent les initiés avec les esprits particuliers qui font d'eux des personnes différentes). À Reite, une personne est donc un processus et une trajectoire, qui subissent des transformations continuelles: naître, grandir et manger du porc, avoir de la peinture appliquée sur le corps, être initié, se marier, obtenir un garamut, et finalement, bien sûr, mourir, moment où la dissolution de la personne est prise en charge par les parents survivant jusqu'à ce que la substance et la nourriture qui l'ont constituée aient été remises en circulation à travers d'autres (Battaglia 1990, Coppet et al. 1994, Leach 2003 : 186-189).

À propos de ces affaires de substitution et de remise en circulation, Douglas Dalton (1996) a écrit de façon intéressante sur le vide émotionnel provoqué par la perte d'une sœur au profit d'un autre groupe de parenté au moment du mariage, ou suivant la mort d'un parent, et sur les paiements compensatoires en parures de coquillage effectués en de telles circonstances. Il se focalise sur ce qu'il appelle la beauté « sublime » des coquillages et des dents de chien (tels ceux que portent Sambong et Tariak sur les Figures 6 et 7 ) des 

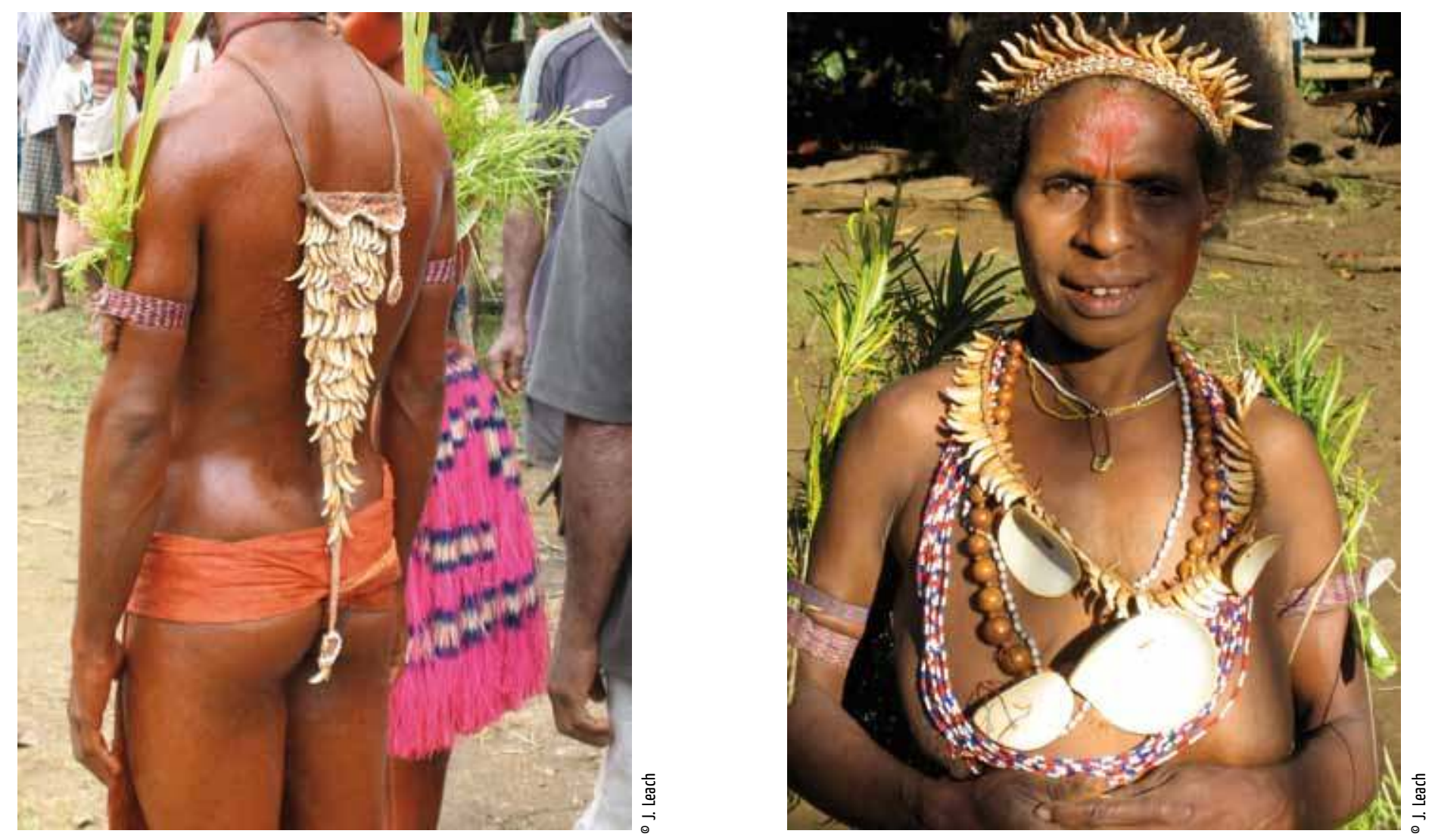

Sambong et Tariak (Fig. 6 et 7)

locuteurs de Rawa (des voisins méridionaux des locuteurs de Nekgini). Il soutient que les coquillages sont « sublimes » aux yeux des Rawa parce qu'ils se substituent à la personne et la remplacent, tout en laissant un vide, tout en laissant la douleur intacte alors qu'ils compensent la perte de la substance que provoquent de tels échanges ou un tel décès, mais la douleur de la perte est intacte. La beauté, ici, est teintée de douleur intense. Néanmoins les personnes, à force d'exister en tant que personnes humaines, sont faites du travail et de la substance des autres. Cette substance et ce travail ne sont jamais qu'empruntés, en quelque sorte. C'est l'aspect matériel de la parenté, parce que son existence provoque toujours une obligation de remise en circulation pour les autres. Et elle peut faire l'objet de substitution, être transformée en objets pouvant être échangés. En ce sens, une personne est remplaçable, étant toujours remplacée et régénérée. L'idée de Dalton est que les ornements de coquillages n'atteignent pas leur objectif de symbolisation de la personne et sont donc à la fois nécessaires et insuffisants pour les Rawa. Je reviendrai plus loin sur l'argument de Dalton.

Ceci dit, personne à Reite ne songerait à déclarer qu'un garamut est identique à un corps de chair et de sang, mais tout le monde est d'accord pour dire qu'il possède une voix et qu'on peut en parler comme d'une personne. Lorsque les habitants de Reite disent qu'un « garamut est un homme », il se peut qu'ils le pensent littéralement, mais alors la question 
devient: de quel aspect d'un homme s'agit-il? Et effectivement, qu'est-ce qu'un homme à Reite? C'est là l'essentiel de cette première partie de mon exposé.

Un garamut n'est pas un objet de richesse substituable parce qu'il est l'apparence que prend une identité à un moment particulier qui ne peut pas être reproduit. Et il en est ainsi (j'hésite, mais suis à présent attaché à le montrer) du fait de sa forme matérielle. Il existe et résulte du fait d'une transformation spécifique et particulière: celle qui confère à un homme une présence audible dans un paysage. Qui modifie des relations avec des affins et des germains pour lui donner une position en tant qu'homme disposant d'une voix: comme quelqu'un à qui des gens vont répondre du fait des obligations qu'ils ont envers lui. Les obligations sont forgées dans l'émergence même de l'objet qui est la manifestation, l'aspect visible et audible de ce processus de transformation matérielle, sociale et spirituelle.

La façon la plus simple d'énoncer cette idée consiste peut-être à dire qu'il est impossible de reproduire un garamut. Chaque nouveau garamut opère une nouvelle transformation des relations entre des germains et entre un homme et ses affins. Il est en fait impossible de fabriquer un autre garamut pour une même personne, car cela ferait d'eux une personne différente: définie par un nouvel ensemble de relations, comme personne et comme objet. La seule fois qu'un garamut est transformé, c'est à la mort de son propriétaire. Dans le passé, une fois la chair disparue, les ossements des hommes étaient posés à l'intérieur de leur garamut, et le tout était placé dans le séjour chaud et sec des esprits, dans les forêts. Personne n'héritait du garamut. J'espère que le lecteur voit pourquoi.

Il n'y a que dans une circonstance extraordinaire, lorsque l'épouse d'un homme meurt et que le veuf prend une nouvelle épouse parmi ses affins, qu'un garamut peut être obsolète. Mais pour qu'un homme de Reite puisse prendre une seconde femme, il doit, dans tous les cas, dissoudre le lien avec ses anciens affins par de grands échanges, ce qui l'affranchit des obligations qu'il a envers eux et rend caduque le garamut qui incarnait leur connexion. Je suggère donc qu'un garamut de Reite est un « objet irremplaçable ».

Je suis intrigué par la symétrie de la perte dans le cas d'une personne et d'un garamut, par le fait que, dans l'analyse de Dalton, remplacer une personne revient à laisser un vide résiduel : «Les locuteurs Rawa reconnaissent explicitement que les kunawo [parures de coquillages] ne peuvent jamais remplacer la personne pour laquelle ils ont été échangés » (Dalton 1996 : 398). En ce sens, les parures de coquillages constituent une sorte d'événement marqueur, de jeton-souvenir (token event), quelque chose qui remplace la chose réelle (la sœur absente). La personne est substituable, mais cela ne signifie pas que son caractère unique et son attachement émotionnel aux autres sont oblitérés par de tels échanges. Dans le cas d'un garamut de Reite, ce sont aussi les relations avec les autres qui font que quelque chose est irremplaçable, mais dans le cas de cet objet, sa forme matérielle, conférée spécifiquement par certaines relations, est la source de sa présence et de son pouvoir, en même temps que celle de son irremplaçabilité: il ne peut pas être ultérieurement retransformé en des relations identiques à celles qui l'ont créé. Ces relations se sont elles aussi transformées, et ne sont donc plus les mêmes. Les garamut sont l'exemple d'une chose prenant les attributs d'une personne, formée au sein de celle-ci et donc considérée comme telle - une personne spécifique dotée d'un genre, qui plus est. Cela ne signifie pas pour autant qu'il y a une substitution globale. Ce qui est intéressant ici (dans la notion d'objets irremplaçables ou uniques), c'est d'insister sur la question de savoir en quoi chaque cas de personne/forme diffère d'un autre, et de comprendre ce que ces différences impliquent. Allons-nous chercher cette différence dans le « matériel »? Quels sont les éléments physiques/matériels qui font que les gens considèrent ces objets comme irremplaçables? 
J'ai déjà fait allusion au fait qu'il semble que le cas des garamut met en cause les fondements même de cette question précise : il n'y a rien ici de « matériel » qui ne soit tellement lié dans sa forme aux relations qui entourent la fabrication, l'usage et la légitimation d'un garamut, qu'il est impossible de le séparer d'eux et de le considérer indépendamment, en quelque sorte. Mais je prends soin de poser la question suivante: qu'est-ce que les habitants de Reite considèrent eux-mêmes comme les « aspects matériels qui rendent les choses irremplaçables »?

Chaque garamut rend visible un moment des relations entre des personnes, leurs esprits, le lieu de sa fabrication, etc. Il opère également une transformation dans ces éléments. Pour cette raison, chaque garamut est différent. Les habitants de Reite insistent sur le fait que la qualité du son d'un garamut repose sur la réalisation correcte des procédures rituelles lors de sa fabrication. Il dépend aussi de l'état moral et spirituel des personnes qui le fabriquent, et de celui qui le reçoit. Le conflit, la rancune, la pollution, etc. donneraient pour résultat un tambour à fente abîmé, ou à faible résonance.

Le tambour fabriqué pour le British Museum (celui que j'ai commandé) s'est révélé doté d'un « visage » ou d'une « tête » qui ressemblait très clairement à un rocher situé dans la demeure d'un esprit, sur les terres d'un de mes voisins. Ce vieil homme s'anima en constatant cette ressemblance, qui avait apparemment surgi spontanément des mains de plusieurs sculpteurs différents. Le fait que ma maison soit située sur ses terres fut longuement discuté, et à un moment, il sembla que le projet tout entier fût menacé par l'apparition du visage de cet esprit sur le garamut du musée. Emporter le garamut que j’avais commandité était une chose. Enlever une manifestation visible d'un puissant ancêtre mythique en était une autre, toute différente. Personne n'était sûr de ce que voulait dire l'émergence de cette forme, mais il était clair pour eux qu'elle disait quelque chose de ma relation à la terre sur laquelle j'habitais, et donc mes obligations envers ses descendants. Finalement, nous avons admis la similarité, nous nous sommes excusés pour sa révélation involontaire, et avons donné des richesses au vieil homme et à ses parents en reconnaissance de l'obligation qui apparaissait clairement dans la forme que le garamut avait prise. Une telle forme exprime l'état des relations entre les parties qui lui ont donné naissance. C'est dans les qualités de l'apparence et de la voix du garamut que les gens eux-mêmes voient un caractère unique.

Nous devons donc en conclure que les garamut sont comme une personne et en diffèrent, dans la mesure où la dernière transformation dans le corps et la personne d'un homme est réalisée par la fabrication du garamut. L'ancrage de la voix dans ce bois taillé particulier est également celui de ses relations avec ses affins, et de son identité, jusqu'à sa mort. L'irremplaçabilité est conférée comme un moment qui ne peut pas être répété.

J'ai suggéré que les garamut sont et ne sont pas comme des personnes, et qu'ils sont irremplaçables d'une manière qui est à la fois semblable et différente de celle dont les personnes le sont (c'est-à-dire que les personnes peuvent en fait être plus facilement extraites de leurs positions relationnelles, et plus facilement transformées au cours de 
leur vie que les garamut, jusqu'à ce la personne en question soit un homme en position de recevoir un garamut). Les garamut sont une image d'un moment de transformation des relations entre affins, et sont donc efficaces lors des transformations continuelles de ces relations. Mais une fois que le moment est passé, le travail de la transformation achevé, ils ne peuvent plus être refaits ni remplacés.

\section{Mort d'un tambour}

Le caractère « irremplaçable » d'un garamut étant établi, je vais à présent raconter l'événement sans précédent que j'ai mentionné plus haut. Cela conduit à la prise en compte de la relation fluctuante que les gens ont avec les garamut sur la Rai Coast.

En 2010, un garamut achevé, qui possédait une voix et était utilisé, fut attaqué. Il fut en fait « tué », selon les dires de la population locale. Ce garamut avait été fabriqué pour l'école locale afin d'appeler les enfants (et d'autres) en classe chaque jour. L'histoire de ce qui amena là ce garamut a son importance.

La première école de la région fut construite à un endroit appelé Lamtub, un village de la côte (situé dans un groupe de langue différent). Depuis sa création pendant la période coloniale, cette école était la plus proche de Reite et, au cours des ans, de nombreux enfants de ce village y étaient allés. Le village de Reite a obtenu sa propre community school en 1991, après de nombreux travaux bénévoles pour dégager la forêt et fabriquer les salles de classe et les habitations des instituteurs avec des matériaux locaux. C'est à cette époque que le Conseil de l'école de Lamtub souhaita avoir un garamut en guise de cloche d'appel pour son école. Ils s'adressèrent aux habitants de Reite, qui sont connus pour leur expertise dans ce domaine. En tant qu'initiatrice et commanditaire de la fabrication, l'école de Lamtub proposa d'offrir la viande requise pour nourrir les esprits et faire des paiements lors de chaque étape de la fabrication (Leach 2002 : 721-725).

La requête pour un garamut fut discutée lors d'une réunion de village à Reite. Un homme du nom de Nim, originaire du hameau de Marpungae, prit la parole pour dire que se trouvait sur ses terres un arbre de bois de fer tombé au sol et qu'il serait heureux d'en faire don. Un représentant du Conseil de l'école de Reite demanda si eux aussi pouvaient disposer d'un garamut qui serait fabriqué en même temps, et deux tambours à fente furent faits à partir de l'arbre de Nim. Mais alors que les commanditaires, les gens de Lamtub, fournirent la majeure partie du paiement pour la fabrication, le Conseil de l'école de Reite n'offrit aucun porc ni aucune sorte de viande. Il donna la somme de 200 kina (l'équivalent de $300 €$ à l'époque) en espèces aux hommes dont les esprits avaient creusé le tronc (ce sont toujours les esprits qui réalisent les transformations en un corps - dans le cas présent celle d'un tronc en une personne dotée d'une voix). Cette somme fut répartie entre ces seuls hommes ( 7 au total). Le garamut, une fois terminé, fut traîné jusqu'à l'école de Reite sans autre cérémonie ni paiement. Près de vingt ans passèrent, au cours desquels ce tambour fut utilisé quotidiennement. Puis, en juillet 2010, dans l'obscurité de ma seconde nuit passée au village, on l'entendit produire un son très inhabituel car quelqu'un le frappa à coups de hache. Il y eut un grand tumulte et beaucoup de monde, dont des gens venant de très loin, se précipita en direction de l'école en traversant notre hameau. 
L'histoire de la dispute derrière cette attaque est longue et compliquée. En bref, un des fils de Nim, un enfant en classe de $4^{\mathrm{e}}$ (grade 8 ) avait une petite amie qui était elle aussi une élève de l'école. Il est contre les règles de l'école que les élèves soient mariés, ou grisim (flirtent en Tok Pisin), pendant le temps qu'ils sont à l'école. Le père de la fille était mécontent de cette liaison et il refusa de payer ses droits d'inscription. «Qu'ils se marient! », déclara-t-il plein de colère et de frustration. Le garçon devint furieux à son tour, à l'idée d'être exclu de l'école s'il était marié. Il dirigea sa colère contre le Conseil de l'école qui, disait-on, était sur le point de faire respecter les règles, ainsi que sur d'autres personnes bien précises qui s'étaient plaintes du « mariage ». Cette nuit-là, il tenta de détruire le garamut de l'école (avec l'aide de frères de Marpungae), ce qui était une façon de montrer son mécontentement. Puis il quitta l'endroit en hâte pour se réfugier dans la famille de sa mère, loin de là et hors d'atteinte.

Les gens ont pleuré le garamut. Lacte de destruction causa une réaction violente et un trouble persistant pendant les semaines que je passais sur place. Ceux qui furent le plus touchés étaient les hommes qui l'avaient fabriqué pour l'école. Ce sont eux qui soulignèrent qu'il s'agissait d'un acte violent, d'une tentative de meurtre, et cherchèrent le châtiment le plus sévère possible. Ce sont ces mêmes hommes qui furent le plus visiblement affectés par le son que produisait à présent le garamut. Ce garamut a eu désormais une étrange sorte de moitié de vie, une vie où sa voix parle de façon éloquente de l'histoire problématique qui l'entourait, des relations avec l'institution de l'école, et de son mode de fabrication.

Rappelons ici que cet événement était totalement inédit. Personne n’avait jamais attaqué le garamut d'autrui. Comment cela avait-il alors pu se produire? Qu'est-ce que le garamut de l'école avait de différent qui ait pu rendre possible une telle démonstration de colère dans cette attaque sans précédent? Il faut évidemment remonter à sa fabrication. C'est là que se manifeste l'effet majeur de reconfiguration par le garamut des relations entre les personnes, relations auxquelles il est conféré une solidité et une persistance temporaire à travers l'apparence de l'objet lui-même.

La fabrication du garamut pour l'école n'avait pas impliqué de lien ou d'interactions ultérieures avec les affins. Il est évident que le type d'entité que représente l'école (une institution externe particulière) autant que la façon dont fut commandé le garamut font que ce dernier était d'un type différent. La relation de l'école vis-à-vis de son tambour n'était pas la même que celle d'un homme avec ses affins et avec son garamut, qui se

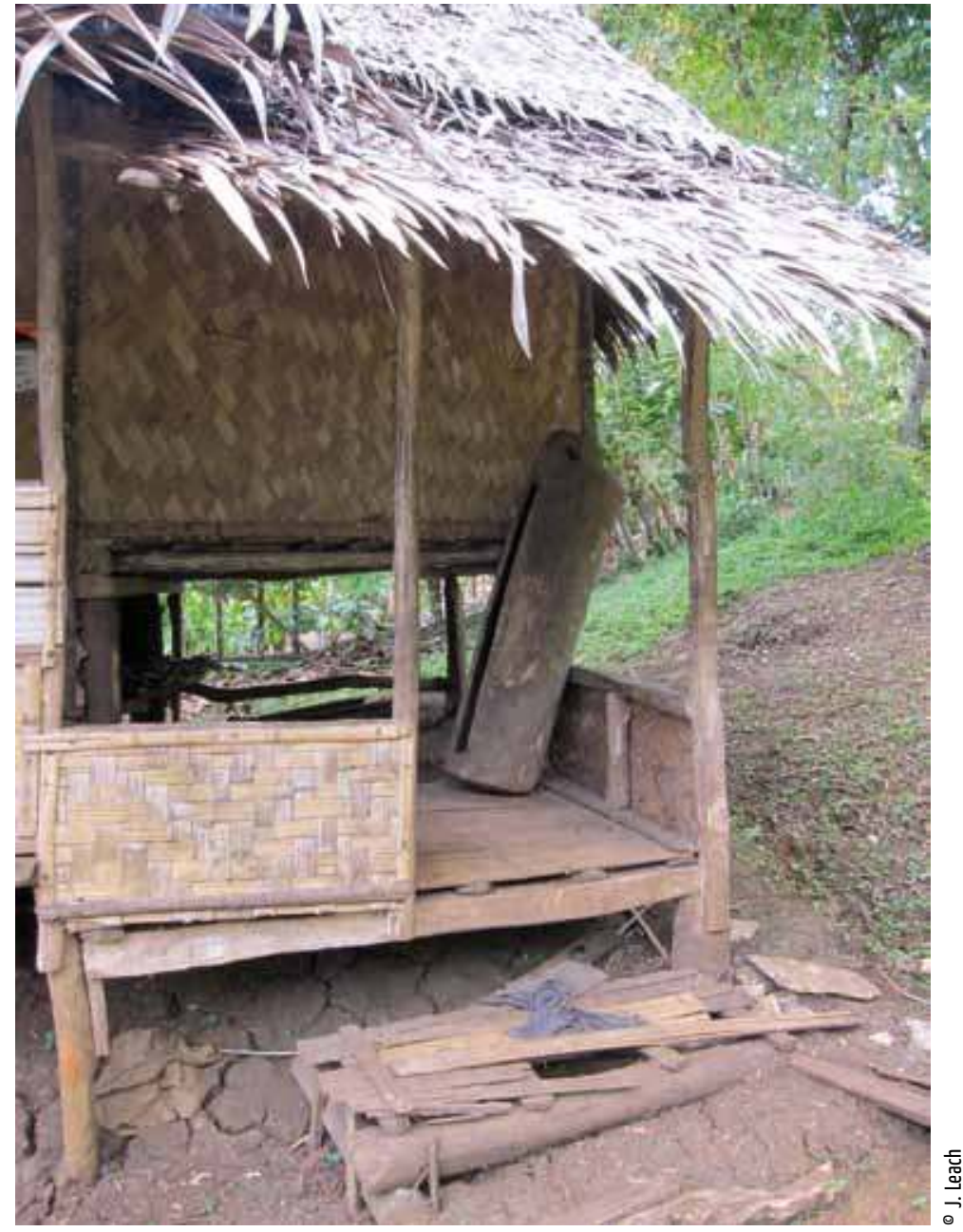

Un Garamut de Reite

Tambours à fente, ignames et effigies. Une excursion dans la substituabilité.

(Fig. 1) 
situe au fondement de l'émergence de celles-ci. Son apparence ne marquait pas une transformation de position et d'autorité, elle ne plaçait l'école dans aucune relation particulière vis-à-vis d'autres personnes spécifiques qui lui seraient obligées (même si il y avait probablement une attente de ce type à Marpungae). Le fait que le garamut ait été fabriqué à partir d'un arbre provenant d'une terre de Marpungae a fait penser que la « personne » de l'école, son identité, était étroitement liée à Marpungae. D’une certaine manière, l'école était davantage celle de Marpungae que celle d'autres gens. En appui de cette idée, il faut dire qu'il y a toujours eu une ambiguité autour du détachement du tambour hors de Marpungae. Les 200 kina n’avaient pas été donnés à Nim ou à d'autres habitants de Marpungae, mais aux hommes qui avaient fabriqué le garamut. La dispute qui causa sa perte concernait le mariage, mais un mariage où l'école était un troisième parti étrange et malvenu. Au lieu de soutenir le garçon impliqué, comme cela aurait été le cas pour le propriétaire d'un garamut fabriqué sur les terres de celui-ci, l'école fut un obstacle à son mariage. Cela signifiait qu'il était justifié, de son point de vue, de détruire l'incarnation de leur lien - puisque, pour lui, ce lien était un mensonge, une farce.

En fait, ce garamut lui-même avait été fabriqué au cours d'une sorte de parodie du processus de fabrication d'un tel objet, même si nombre des éléments de ce processus furent présents d'une façon ou d'une autre. (En l'occurrence, l'école possède une autorité et peut déplacer les gens [les enfants, leurs parents] par sa volition [sa voix] une fois dotée d'un garamut. Lambtub était l'aîné des germains et a initié la fabrication, qui a été entreprise " pour la communauté » et ce sont eux qui allaient entendre la voix du garamut etc.). Mais au lieu que cette obligation soit générée par une transformation spécifique de personnes particulières, liée à l'apparence matérielle d'un objet unique envers lequel ils avaient des obligations, l'autorité de l'école était diffuse, elle concernait une « communauté » souhaitant voir ses enfants bénéficier d'une éducation comme s'ils étaient tous semblables (et non pas relevant de relations hiérarchiques particulières entre germains, ou de relations entre affines). Il y a donc eu une confusion, ou bien des perceptions différentes de ce que l'école était et de ce qu'elle est, et de la façon dont le garamut participe à sa présence. D'où la confusion concernant la relation que le garamut exprimait et opérait.

On notera ici une autre confusion: l'école considérait clairement et de manière compréhensible que le garamut lui appartenait. (Elle l'avait acheté, et les enseignants, venant souvent d'autres régions de la Nouvelle-Guinée, l'utilisaient comme une simple cloche pour appeler les élèves de manière indifférenciée, c'est-à-dire sans référence à aucune relation de construction mutuelle). Pour l'école, le garamut était un objet reproductible ou remplaçable, exactement comme le seraient d'autres types d'objets que l'on peut acheter. Sa perte mit ses membres en colère, et ils exigèrent son remboursement auprès des gens de Marpungae, mais, contrairement à de nombreuses personnes de Reite, les instituteurs de l'école et la police qui vint de loin pour juger l'affaire ne considérèrent pas l'attaque comme quelque chose de particulièrement choquant. Ils traitèrent la question comme une destruction de biens personnels.

Or, pour les gens de Marpungae, le garamut n'était pas un simple bien, une simple possession. Il rendait visible une obligation non remplie que l'école avait envers eux. Le choix du garamut comme exutoire à leurs revendications fit apparaître au grand jour le caractère spécial de leur relation à l'école. Ils agirent comme ils le firent car ils avaient, vis-à-vis du garamut, un intérêt que l'école ne pouvait pas reconnaître. Toute la différence, le point crucial, est que les relations d'obligation et de construction mutuelle inhérentes à la fabrication d'un tambour à fente étaient transformées en quelque chose qui ressemblait à un droit de propriété exclusif sur un objet. 
En fait, comme je l'ai déjà dit, les distinctions matérielles et sociales se confondent dans le garamut, et nous voyons ici comment la séparation du social et du matériel s'opère à Reite. Le garamut est la propriété de l'école, elle a des droits sur celui-ci en raison de son achat. Mais ce n'est pas pareil qu'avoir des relations avec les autres qui passent par des obligations rendues visibles et effectives par la chose elle-même. Une abstraction de la relation est advenue: ce qui, pour d'autres garamut, est constitutif - l'obligation vis-à-vis d'autres personnes - est dans ce cas, un droit de propriété sur un objet en tant que tel.

Il a été suggéré par Eduardo Viveiros de Castro que les droits sont le corollaire relationnel du don dans une économie de biens (2009 : 250-251). Les droits incarnent la façon dont les relations sont rendues visibles. Non pas à travers le mouvement des objets, mais sous leur forme abstraite dans les droits que détient la personne. Je pense que nous devons envisager l'attaque contre le tambour comme un aspect de l'émergence de cette abstraction à Reite au cours des vingt dernières années, et donc, peut-être, comme ce qui constitua la véritable mort du garamut: il n'avait plus de raison d'exister.

\section{Représentation, affect et formes génériques}

Qu'est-ce qui rend le passage au générique possible? Quels sont les bouleversements historiques et les conditions sociales qui rendent possible la copie d'un objet, d'en fabriquer un qui ne soit pas unique? Pensons à une version d'une connexion abstraite par le biais des droits légaux: la loi du copyright, par exemple. Un copyright est le droit que possède un individu sur ses créations individuelles, en tant qu'elles sont le produit de son propre travail (mental). L'expression est logiquement séparée de son créateur - elle est exprimée! L'objet est ensuite lié à la personne du créateur par le biais d'un mécanisme légal qui recrée, sous la forme abstraite d'un « droit », un lien qui était auparavant interne à la personne. Nous pouvons donc dire que, selon la loi sur le copyright, une liaison physique associée à une idée est remplacée par un droit sur un objet, une fois que l'objet advient en tant que tel. Cette abstraction d'une connexion en un droit a de multiples permutations et effets: l'un d'entre eux est que les droits de copier une expression peuvent être échangés, peuvent être enfreints, etc.

Le garamut dont nous parlons ici n'existe pas au sein de tels régimes de droits sur des objets. Le garamut n'est pas une création individuelle. Il est une manifestation, sous forme physique, d'une transformation de personnes qui requiert la présence de celles-ci pour avoir un effet. Il a sa réalité et ses effets dans ces personnes. Les gens ont des obligations les uns envers les autres du fait de sa fabrication, et ces obligations et relations sont rendues présentes chaque fois qu'il résonne. J'ai tenté de montrer pourquoi, pour cette raison, il est impossible de produire une version générique d'un garamut. Chacun d'entre eux correspond à une transformation unique.

Pourtant, l'école possédait un garamut, comme s'il était possible de fabriquer un garamut générique: quelque chose qui ressemble à un garamut et produise le même son, mais n'en est pas un. La cloche de l'école ne fonctionnait pas de la même façon, elle n'établit pas la connexion de relations entre des personnes comme ce serait le cas pour un garamut. C'est cela qui a rendu le garamut de l'école « inadéquatement approprié » à son attaque, parce que même si cette attaque visait des personnes (le conseil de l'école, l'image de la 
communauté), ce sont les droits que les gens avaient sur le garamut qui ont été violés, et non pas un ensemble physique ni un corps. Le garamut de l'école était un simulacre, une copie générique, comme s'il avait été possible de copier un garamut, comme si un tel objet pouvait exister dans un régime d'expressions et de droits individuels sur les objets.

La relation d'un homme à son garamut ne consiste pas en un droit sur une expression. C'est une relation de construction mutuelle, qui l'attache expressément aux relations qui sont exactement celles qui lui donnent une forme particulière d'homme doté d'une voix. Le garamut de chaque homme est irremplaçable. Ou plutôt, dans le système au sein duquel fonctionnent les garamut, les recréer est chose impossible. Le cas de l'école montre un éloignement vis-à-vis de ce système: un acte inédit et impensable est devenu possible soit parce que la situation socio-économique de la Rai Coast évolue, soit parce que le tambour à fente concerné n'en était pas vraiment un, ou plus précisément, du fait d'un mélange de ces deux raisons. Les garçons de Marpungae ont enfreint des principes moraux pris pour acquis parce que l'époque l'a permis. Avec l'arrivée des institutions et d'autres formes d'activité économique les circonstances ont changé. Ces circonstances sont rendues apparentes comme une partie de l'imbrication des personnes et des choses, et de la forme même de ces éléments - leur forme matérielle manifestant ou rendant visible certaines relations. Il s'est avéré possible d'attaquer la cloche de l'école parce que celle-ci avait une position ambiguë, position issue d'un type nouveau de fabrication et d'usage.

Le fait est qu'il est bien sûr possible de remplacer un garamut, mais le nouveau ne sera pas la même chose, tant au sens propre que dans un sens plus général. Un garamut est unique parce qu'il opère une transformation particulière des relations entre les personnes, puis participe à leur stabilisation sur une période donnée. Sa forme stable - matérielle signifie deux choses: on ne peut pas le transformer davantage (contrairement aux corps des êtres humains), ce qui est alors aussi le cas de son propriétaire, qui devient différent des autres corps humains dans sa relation à d'autres personnes précises, des affins et des germains, qui demeurent stable jusqu'à sa mort.

Douglas Dalton a écrit de façon convaincante à propos du « caractère symbolique d'images de coquillages pour transmettre les concepts idéaux qu'ils incarnent » (1996: 394). Il lie explicitement cette perception d'inadéquation à la violence et aux bouleversements coloniaux - c'est-à-dire les nouvelles institutions et la nouvelle économie. Il nous dit que les « kunawo marquent l'absence des individus pour lesquels ils ont été échangés par le fait d'être associés à leur séparation et à leur "mort" » (1996: 399). En tant que « jeton » associé à un événement, en tant que représentation d'une personne sous une autre forme, ils portent en eux un affect et symbolisent la perte, autant que la possibilité d'échange et de compensation. Il semble alors raisonnable de considérer les kunawo comme "présence absente », ou comme représentation d'une personne qui ne peut être retrouvée. L'affect, le chagrin et les sentiments de perte en sont le résultat, aussi «magnifiques » puissent-ils être.

La même inadéquation pourrait être attribuée au garamut de l'école au sens où celui-ci n’aurait jamais pu jouer le rôle d'un vrai garamut. Les actes des jeunes garçons de Marpungae reflètent cette inadéquation. Il s'agit d'une inadéquation d'effet (du tambour) due à l'incomplétude de sa réalisation. Le garamut de l'école n'aurait jamais pu participer activement au déploiement unique et reproducteur des relations entre affins. Dalton nous prévient de l'inadéquation des objets à remplacer effectivement les personnes. Mais son analyse concerne les objets symboliques. Le problème avec le transfert sans nuance de l'analyse de Dalton aux garamut de Reite est son affirmation que les coquillages de valeur représentent 
les personnes qu'ils remplacent, ce qui n'est pas le cas des garamut. Tels que Dalton les décrit, les kunawo diffèrent totalement des garamut sur ce point. Ils ne sont pas créés dans et par les mêmes séquences que celles qui lient les gens entre eux d'une façon unique. Le garamut n'est pas une représentation d'un homme ou de sa voix. Il est la relation entre affins, la voix de l'homme etc., et occupe une position relationnelle en tant que tel. Dans sa capacité à faire (effectiveness), il est constitué par et dans ces relations, et n'est donc pas la représentation de quelque chose qui, sans quoi, serait tout à fait absent. Il est la chose elle-même.

Le chagrin qu'ont ressenti les gens à l'attaque du garamut de l'école était davantage semblable à l'affect associé à un objet symbolique - en fait, cet « affect » est ce que ressentent les gens à proximité des objets qui évoquent la mémoire par le biais d'une association avec une disparition passée ou d'une représentation de celle-ci. Mais hormis le cas de celui de l'école, l'irremplaçabilité des garamut n'a rien à voir avec la représentation ou l'affect. Elle a à voir avec leur participation active dans la genèse et l'entretien de formes de relations particulières entre les personnes. Un garamut ancien et brisé après la mort de son propriétaire ne porte pas en lui d'affect, mais plutôt du respect mêlé de crainte. Il contient la transformation ultime de son propriétaire en des ossements chauds, secs et puissants, qui confèrent un pouvoir ancestral et spirituel aux terres sur lesquelles ils sont conservés. On pourrait suivre Dalton en allant aussi loin que dire que les objets portent en eux l'affect et le suscitent. Ce sont des « jetons » renvoyant à des événements d'un autre temps et d'un autre lieu, qui ne remplacent jamais la « vraie » chose. Leur « agentivité » est d'un type secondaire plutôt que primaire, c'est une agentivité qui passe par un objet (mediated). Mais ce n'est pas là une description adéquate pour des choses de la Rai Coat. Les garamut ne sont pas des objets au sens où Gell l'entend lorsqu'il présente les œuvres d'art comme étant des index de l'agentivité de leur créateur. Les garamut constituent des moments émergents dans les relations qui n'appartiennent à aucun groupe ou aucune personne. Ils appartiennent à la relation entre affins et sont cette relation elle-même. J'ai ainsi tenté de montrer dans ces pages un caractère temporel et relationnel de l'irremplaçabilité, plutôt qu'un aspect essentiel ou symbolique. 


\section{NOTES}

Photo d'ouverture: Garamut brisé, 2010

1. Je dois cette expression inappropriately inappropriate à Kath Weston.

\section{RÉFÉRENCES}

Battaglia, D. 1990 On the Bones of the serpent: person, memory, and mortality in Sabarl Island Society. Chicago: University of Chicago Press.

Biersack, A. 1982. Ginger gardens for the Ginger woman: Rites and passages in a Melanesian Society. Man, n.s. $17: 239-58$.

Burridge, K. 1960. Mambu: A Melanesian millennium. London: Methuen.

Coupaye, L. 2009 What's the matter with technology? Long (and short yams), materialisation, and technology in Nyamikum village, Maprik district, Papua New Guinea. TAJA (20) : 93-111.

de Coppet, D. , Barraud, C., Iteanu, A. \& Jamous, R. (dir.) 1994 Of Relations and the dead: four societies viewed from the angle of their exchanges (Traduit par S. Suffern). Oxford: Berg.

Dalton, D. M. 1996 The Aesthetic of the sublime: an interpretation of Rawashell valuable symbolism, American Ethnologist 23 (2): 393-415.

Damon, F.H. 1980 The Kula and generalised exchange. Considering some unconsidered aspects of the elementary structures of kinship, Man (NS) 15 (2) : 262-92.

Fortune, R. 1932 Sorcerers of Dobu. New York: E.P. Dutton and co. Inc.

Foster, R. J. 1995 Social Reproduction and History in Melanesia: Mortuary Ritual, Gift-Exchange, and Custom in the Tanga Islands. Cambridge: Cambridge University Press.

Gell, A. 1998 Art and agency: an anthropological theory. Oxford: Oxford University Press.

Gudeman, S. 1986 Economics as Culture. Metaphors and models of livelihood. London: Routledge and Kegan Paul.

Lawrence, P. 1964 Road belong cargo. Melbourne: Oxford University Press.

Leach, J. 2002 Drum and voice. Aesthetics and social process on the Rai Coast of Papua New Guinea, Journal of the Royal Anthropological Institute (N.S.) 8: 713-734.

- 2003 Creative land. Place and procreation on the Rai Coast of Papua New Guinea. Oxford and New York: Berghahn Books

— 2007 Differentiation and encompassment: a critique of Alfred Gell's theory of creativity. In A. Henare, Holbraadand M. \& S. Wastell Thinking Through Things. London: Routledge.

- 2011 Twenty Toea has no power any more: property, customary tenure, and pressure on land near the Ramu Nickel Project Area, Madang, PNG. Pacific Studies 34 (2/3) : 295-322.

Strathern, M. 1979 The Self in self-decoration. Oceania 44 : 241-57 (254).

- 1988 The Gender of the gift. Berkeley: University of California Press.

- 1995 Shifting contexts : Transformations in Anthropological Knowledge. London : Routledge : 17.

- 2005 The Relation. Issues in Complexity and Scale. Cambridge: Prickly Pear Press. ISSN 1351-7961

Viveiros de Castro, E. 2009 Métaphysiques cannibales, Paris, PUF : 250-251.

Weiner, A. 1992 Inalienable possessions. The paradox of keeping-while giving. Berkeley: University of California Press.

Wurm, S.A., Hattori, S. 1983. Language Atlas of the Pacific Area, part 1 \& 2. Canberra, Pacific Linguistics, ANU. 


\section{RÉSUMÉ}

La mort du tambour. Choses uniques sur la Rai Coast de Papouasie-Nouvelle-Guinée. Fabriqués et utilisés dans le cadre des relations d'échanges entre affins de la Rai Coast de Papouasie-Nouvelle-Guinée, les tambours à fente sont réputés avoir une "voix», et donc porter en eux une certaine gravité ; ils exigent du respect, comme s'ils étaient des sortes de personnes. En outre, ils sont si étroitement liés à la personne de leur propriétaire, ainsi qu’à son rang social, que l'on dit qu'ils sont leur voix. Ces objets ne peuvent être extraits de l'unité de parenté où ils sont apparus, et au sein de laquelle ils jouent un rôle continu. Cependant, en 2010, lors d'un événement sans précédent, un grand tambour à fente fabriqué pour et utilisé par une école communale a été attaqué lors d'un conflit. Je me penche ici sur la notion d'irremplaçabilité et de substituabilité à la lumière de cet incident. Je suggère en conclusion de l'article que les tambours à fente de la Rai Coast ne sont pas des représentations de personnes, mais des choses uniques et spécifiques en soi, en raison de la position « relationnelle » qu'ils occupent. Ils ont en cela un caractère unique temporel plutôt que lié à leur essence.

\section{ABSTRACT}

The death of the drum. Unique things on the Rai Coast of Papua New Guinea. Slit-gong drums, made and used as part of affinal exchange relations on the Rai Coast of Papua New Guinea, are said to have a "voice" and thus carry gravitas; they demand respect as a kind of person. Further, they are so closely tied to the person and position of their owners that they are said to be their voice. These items cannot be extracted from the kin formations in which they came into being, and in which they have their on-going effect. However, in 2010, in an unprecedented event, a large slit-gong made for and used by a local Community School was attacked during a dispute. I examine the notion of irreplaceability and substitutability in the light of this incident. The paper concludes with the proposition that Rai Coast slit-gong are not representations of persons, but are specific and unique things in their own right because of the "relational" position they sustain. In this, they have a temporal rather than essential uniqueness.

\section{MOTS-CLÉS}

Tambour à fente, personnalité, substituabilité, générique, représentation, mort, affect, Papouasie-Nouvelle-Guinée.

\section{KEYWORDS}

Slit-gong, personhood, substitutability, generic, representation, death, affect, Papua New Guinea. 